\title{
Parathyroid Adenoma Presenting as Acute Pancreatitis
}

\author{
Shaoni D Sanyal ${ }^{1}$, Safika Zaman ${ }^{2}$, Ajitesh Roy ${ }^{3}$, Ranjan Raychowdhury ${ }^{4}$
}

\begin{abstract}
Aim: To discuss an unusual presentation of parathyroid adenoma with management.

Background: Primary hyperparathyroidism (PHPT) is characterized by hypercalcemia with elevated levels of parathyroid hormone (PTH). Parathyroid adenoma is the underlying cause in $85 \%$ of patients with PHPT.

Case description: A 49-year-old woman presented with acute abdominal pain and was diagnosed to have acute pancreatitis. Routine investigations revealed hypercalcemia (serum calcium $>14 \mathrm{mg} / \mathrm{dL}$ ) and elevated PTH $(1,440 \mathrm{pg} / \mathrm{mL}$ ). Initial ultrasound imaging of the neck showed an ill-defined hypoechoic space-occupying lesion with a cystic component in the lower pole of the right lobe of the thyroid gland. This was followed by a Sestamibi scan and SPECT CT. The patient underwent surgery after proper preoperative preparation. The level of PTH was reduced to $293 \mathrm{pg} / \mathrm{mL}$ on the first postoperative day. Serum calcium measured remained within normal limits.

Conclusion: Pancreatitis is a rare presentation of parathyroid adenoma, but the diagnosis must be kept in mind while treating patients.

Clinical significance: Patients with recurrent unexplainable pancreatitis should be worked up for parathyroid adenoma.

Keywords: Gallstones, Hypercalcemia, Parathyroid adenoma, Parathyroid neoplasms, Primary hyperparathyroidism.

World Journal of Endocrine Surgery (2021): 10.5005/jp-journals-10002-1318
\end{abstract}

\section{BACKGROUND}

Primary hyperparathyroidism (PHPT) is characterized by hypercalcemia with elevated levels of parathyroid hormone (PTH). ${ }^{1}$ It is usually seen in females above the age of 50 years, with a prevalence of 21/1,000 in Europe. ${ }^{2}$

The prevalence of the condition in the United States has been estimated at 23 cases per 10,000 women and 8.5 per 10,000 men. ${ }^{3}$

Parathyroid adenoma is the underlying cause in $85 \%$ of patients with PHPT; multiple-gland hyperplasia accounted for approximately $6-10 \%$ of cases; however, parathyroid malignancies are rare, occurring in $<0.5 \%$ of all cases.

The clinical profile of PHPT is usually variable. Patients may present with a classic skeletal disease, recurrent nephrolithiasis, or be asymptomatic, detected on routine biochemical screening. In developing countries, they usually present with overt manifestations.

Data collected from the Indian PHPT registry between July 2005 and June 2015 recorded a total of 464 subjects (137 men and 327 women) with histopathologically proven PHPT.

The mean age of patients was $41 \pm 14$ years with a female:male ratio of 2.4:1. The majority (95\%) of patients were symptomatic. Common clinical manifestations were weakness and fatigability (58.7\%), bone pain (56\%), and renal stone disease $(31 \%)$. Pancreatitis was a rare presentation, occurring in only $12.3 \%$ of the patients. ${ }^{4}$

\section{Case Description}

A 49-year-old woman presented with acute abdominal pain and was diagnosed to have acute pancreatitis. She also had a history of body pains, acid peptic disorder, and episodic depression for the previous 5 years. The patient had suffered a similar episode a year ago which was diagnosed as gallstone pancreatitis at the time. She underwent cholecystectomy for the same. On examination, her findings were consistent with that of acute pancreatitis, with
1,2,4 Department of ENT Head and Neck Surgery, Vivekananda Institute of Medical Sciences, Kolkata, West Bengal, India

${ }^{3}$ Department of Endocrinology, Vivekananda Institute of Medical Sciences, Kolkata, West Bengal, India

Corresponding Author: Shaoni D Sanyal, Department of ENT Head and Neck Surgery, Vivekananda Institute of Medical Sciences, Kolkata, West Bengal, India, Phone: +919051040747, e-mail: shaoni.sanyal@ gmail.com

How to cite this article: Sanyal SD, Zaman S, Roy A, et al. Parathyroid Adenoma Presenting as Acute Pancreatitis. World J Endoc Surg 2021;13(1):32-34.

Source of support: Nil

Conflict of interest: None

tachycardia and abdominal tenderness in the epigastric region. Examination of the neck was unremarkable.

Routine investigations revealed hypercalcemia (serum calcium $>14 \mathrm{mg} / \mathrm{dL}$ ) and elevated serum lipase and amylase (412 and 828 $\mathrm{U} / \mathrm{L}$, respectively). Parathyroid hormone level was found to be 1,440 $\mathrm{pg} / \mathrm{mL}$. DEXA Scan of the patient showed that she was suffering from osteoporosis ( $T$ score $<-3.5$ ).

Initial ultrasound imaging of the neck showed an ill-defined hypoechoic space-occupying lesion with a cystic component in the lower pole of the right lobe of the thyroid gland. This was followed by a Sestamibi scan and SPECT CT (Fig. 1).

The patient underwent surgery after proper preoperative preparation. The lesion was removed after the frozen section affirmed it to be parathyroid tissue (Fig. 2) and the final histopathology report confirmed cystic parathyroid adenoma (Fig. 3).

Immediate preoperative serum PTH was $924 \mathrm{pg} / \mathrm{mL}$ and serum calcium was $9.6 \mathrm{mg} / \mathrm{dL}$. The level of PTH was reduced to $293 \mathrm{pg} / \mathrm{mL}$ on the first postoperative day. Serum calcium measured remained within normal limits. There were no postoperative complications. The patient is currently on follow-up and has had no further 


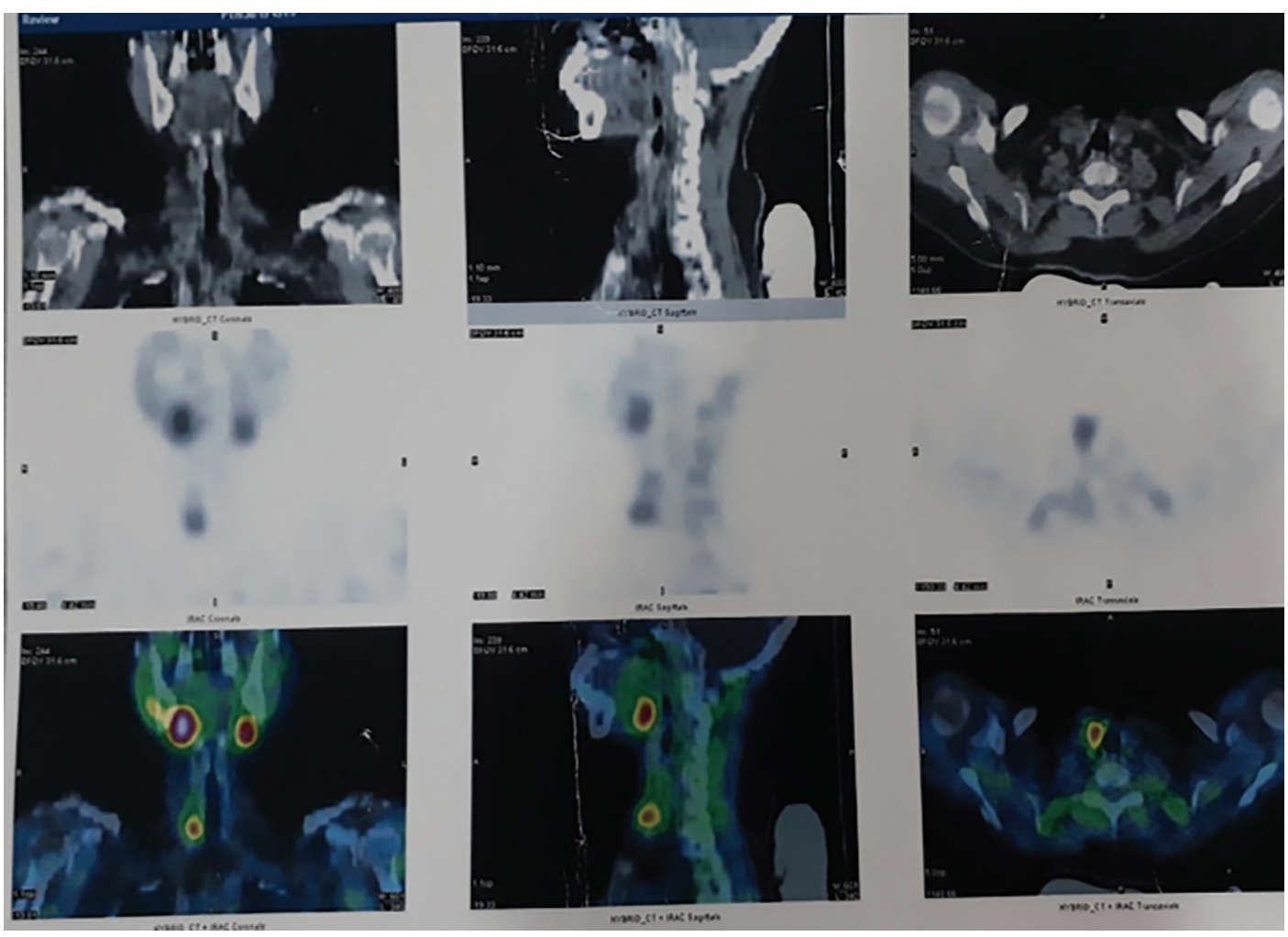

Fig. 1: The 2-hour delayed images on SPECT CT showed an oval-shaped soft tissue density measuring $1.4 \times 1.6 \times 1.7 \mathrm{~cm}$ located posteroinferior to the lower pole of the right lobe of the thyroid gland with increased tracer uptake

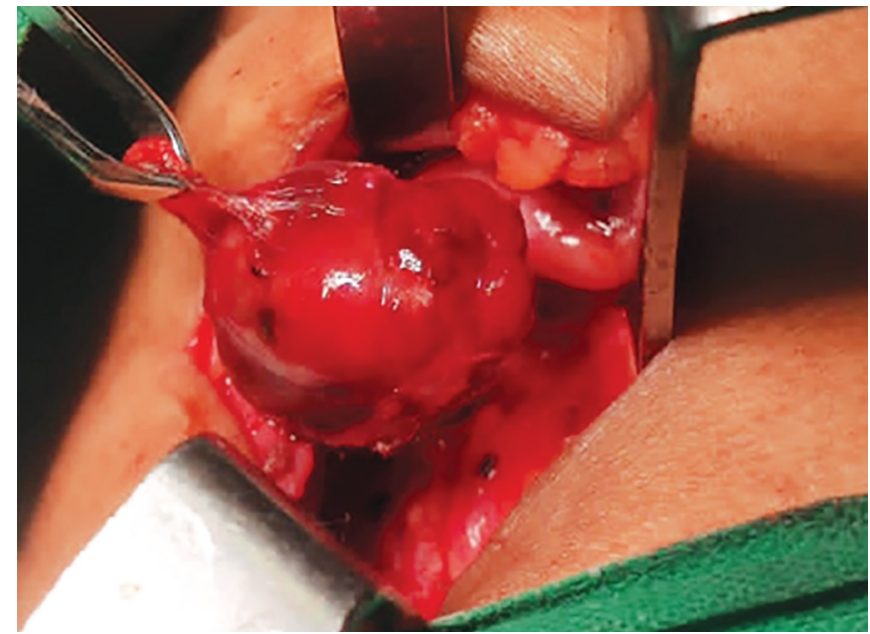

Fig. 2: Right inferior parathyroid gland being removed

episodes of pancreatitis. Thus, surgical excision of the adenoma is curative.

\section{Discussion}

Primary hyperparathyroidism due to parathyroid adenoma presenting as pancreatitis is rare. Its prevalence in the literature ranges from 1.5 to $13 \% .^{5}$

Bess et al. in 1980 described their experience of 1,153 patients with PHPT who presented to the Mato Clinic between 1975 and 1980. Only $17(1.5 \%)$ had coexisting pancreatitis. Usually, the decrease of serum calcium concentration in pancreatitis parallels

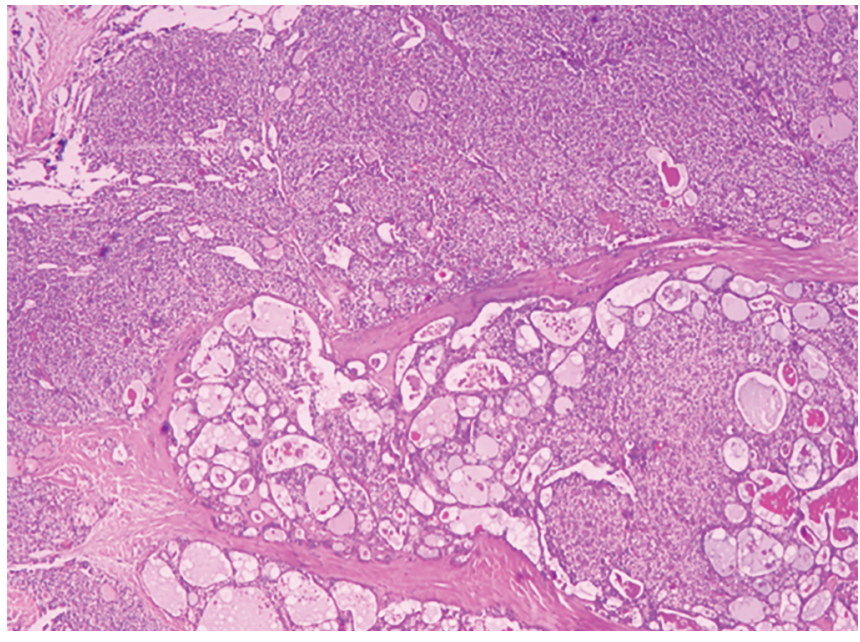

Fig. 3: Histological features: parathyroid showing variable size cystic areas. Fibrous septa intersecting and encircling the sheets of cells. Stain: hematoxylin and eosin, low magnification

the severity of the acute process. If the serum calcium is normal or raised, as was in our case hyperparathyroidism may be suspected. ${ }^{6}$

A retrospective study by Aslam et al. found parathyroid adenoma in $1.94 \%$ of 3,962 patients who presented at the Asian Institute of Gastroenterology with recurrent acute and chronic pancreatitis. The left inferior parathyroid gland was most commonly affected. $^{5}$

Of the 464 patients in the Indian PHPT registry gallstone disease and pancreatitis were documented in 11.1 and $12.3 \%$ of patients, 
respectively. Hypercalcemia was significantly high in PHPT patients with pancreatitis $(12.54 \pm 1.9 \mathrm{mg} / \mathrm{dL})$. Ultrasound of neck and Sestamibi scan successfully localized the abnormal parathyroid gland in 71 and $75 \%$ of patients, respectively. ${ }^{7}$

In our case, the patient had symptoms of bone pain, malaise, and body ache with pancreatitis, but no evidence of pathological fractures. She also experienced depression but this was not formally evaluated. At presentation, her serum calcium was $>14 \mathrm{mg} / \mathrm{dL}$ and the parathyroid adenoma was localized to the right inferior parathyroid gland; whereas previous studies mention the left inferior parathyroid gland to be commonly affected.

There are three possible mechanisms whereby hyperparathyroidism might cause pancreatic damage. First due to necrosis of pancreatic parenchyma by excess PTH. Second, precipitation of calcium in pancreatic ducts causing ductal obstruction leads to secondary pancreatitis. Lastly, increased concentration of ionic calcium in serum leads to an excess of calcium ions in pancreatic juice, which in turn accelerates the conversion of inactive trypsinogen to active trypsin, resulting in pancreatitis. ${ }^{6}$

Felderbauer et al. observed that the occurrence of pancreatitis in PHPT is associated with genetic risk factors such as mutations in the SPINK1 and CFTR genes. ${ }^{8}$

Surgery remains the only definitive treatment for symptomatic hyperparathyroidism. Guidelines from the 4th International Workshop recommend surgery for asymptomatic patients whose serum calcium level is $>1.0 \mathrm{mg} / \mathrm{dL}(0.24 \mathrm{mmol} / \mathrm{L})$ above the upper limit of the normal range, for patients younger than 50 years of age, and for those who have $T$ scores of -2.5 or lower at a central bone densitometry site or in the distal third of the radius or who recently have had a fragility fracture. A glomerular filtration rate of $<60 \mathrm{~mL} /$ minute, renal stones, and a urine calcium level of $>400 \mathrm{mg} /$ day $(10.0 \mathrm{mmol} / \mathrm{day})$ are each considered indications for surgery. The American Association of Endocrine Surgeons also recommends surgery for hyperparathyroidism accompanied by neurocognitive or neuropsychiatric findings. Parathyroid surgery may or may not improve cardiovascular function. ${ }^{3}$

\section{Conclusion}

Pancreatitis is a rare presentation of parathyroid adenoma, but the diagnosis must be kept in mind while treating patients.

\section{Clinical Significance}

Patients with recurrent unexplainable pancreatitis should be worked up for parathyroid adenoma.

\section{ACKnOWLedgments}

The Secretary, Ramakrishna Mission Seva Pratishthan, Kolkata, for the use of Hospital data.

\section{References}

1. Fraser WD. Hyperparathyroidism. Lancet 2009;374(9684):145-158. DOI: 10.1016/S0140-6736(09)60507-9.

2. Adami S, Marcocci C, Gatti D. Epidemiology of primary hyperparathyroidism in Europe. J Bone Miner Res 2002;17:N18-N23.

3. Insogna KL. Primary hyperparathyroidism. N Eng J Med 2018;379(11):1050-1059. DOI: 10.1056/NEJMcp1714213.

4. Bhadada SK, Arya AK, Mukhopadhyay S, et al. Primary hyperparathyroidism: insights from the Indian PHPT registry. J Bone Min Metabol 2018;36(2):238-245. DOI: 10.1007/s00774-017-0833-8.

5. Aslam M, Talukdar R, Jagtap N, et al. Clinical profile and outcome of parathyroid adenoma-associated pancreatitis. Saudi J Med Med Sci 2018;6(2):95. DOI: 10.4103/sjmms.sjmms_80_17.

6. Bess MA, Edis AJ, van Heerden JA. Hyperparathyroidism and pancreatitis: chance or a causal association. JAMA 1980;243(3):246247. DOI: 10.1001/jama.1980.03300290028015.

7. Kearns AE, Thompson GB. Medical and surgical management of hyperparathyroidism. In Mayo Clinic proceedings, vol. 77, No. 1. Elsevier; 2002. pp. 87-91.

8. Felderbauer $\mathrm{P}$, Karakas $\mathrm{E}$, Fendrich $\mathrm{V}$, et al. Pancreatitis risk in primary hyperparathyroidism: relation to mutations in the: SPINK1: trypsin inhibitor (N34S) and the cystic fibrosis gene. Am J Gastroenterol 2008;103(2):368-374. DOI: 10.1111/j.1572-0241.2007.01695.x. 